\title{
B decays to Charmonium at LHCb
}

\author{
Ivan Polyakov
}

ITEP

on behalf of the LHCb collaboration

$16^{\text {th }}$ Lomonosov conference, 27 August 2013 


\section{Outline}

- $\mathrm{LHCb}$ detector + B selection

- $\mathrm{B}^{0} \rightarrow \mathrm{J} / \Psi \mathrm{K}^{* 0}$ angular analysis

- $\mathrm{B}_{(\mathrm{s})}^{0}$ decays to higher charmonium states $\left(\psi(2 S), \chi_{c}\right)$ and neutrals

- X(3872) quantum numbers

- $\mathrm{B}_{\mathrm{c}}^{+}$decays

- Summary 
Features relevant for $\mathrm{B} \rightarrow \mathrm{J} / \Psi \mathrm{X}$

- High efficiency single and di-muon trigger

- Good time resolution to select B candidates

- Good muon identification

- Good K/T separation by RICH detectors

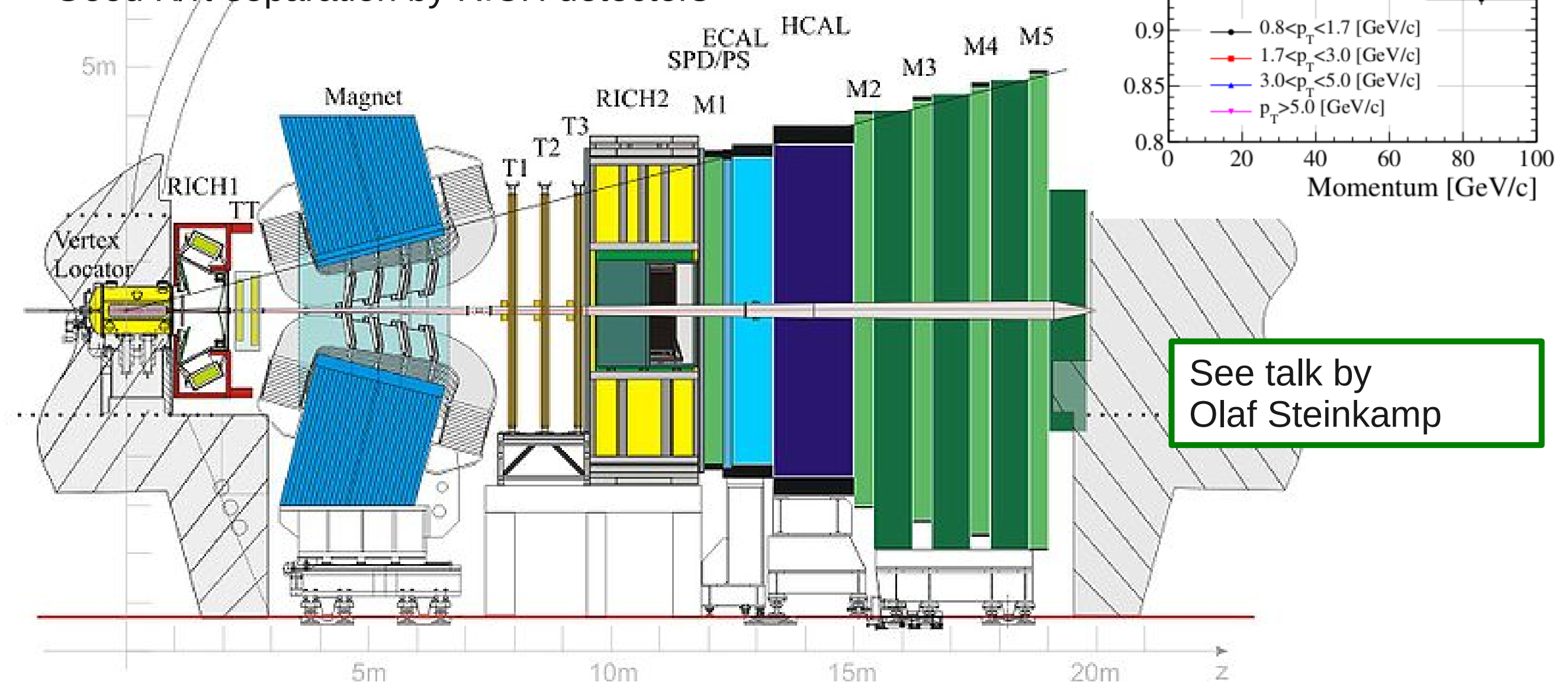


- Trigger on detached $\psi \rightarrow \mu \mu$

- Good quality tracks

- $\quad \mu, \mathrm{K}, \pi$ identification (Muon \& RICH)

- Vertex quality

- PV and SV separation $(c \tau>x)$
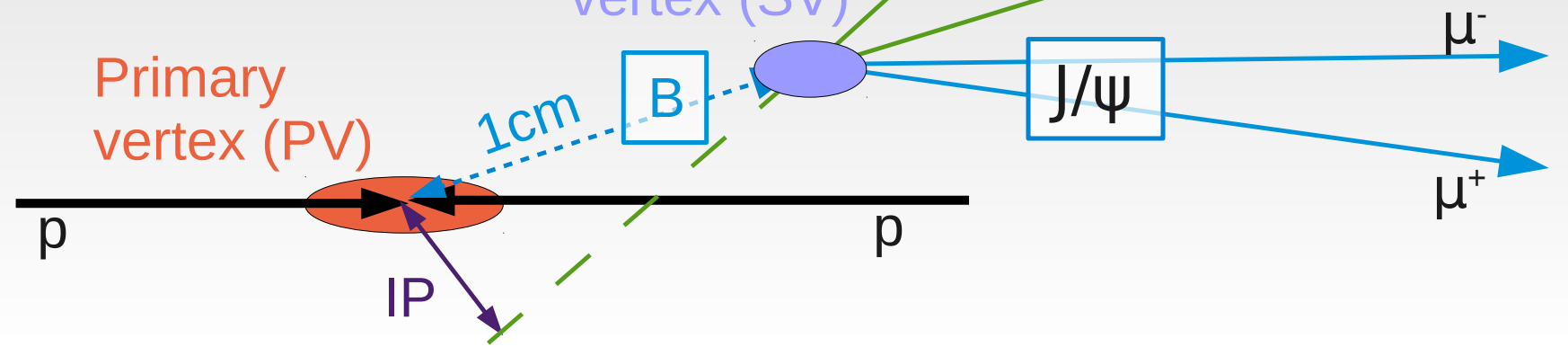

- Daugther particles not from PV

(cut on IP $>2 \sigma, p_{T}>x$ )

- B-candidate from the PV

- Decay structure consistent

Efficiencies and background:

- Efficiencies from simulation

(when possible from data - for PID, trigger)

- use sPlot technique to subtract backgound 


\section{Angular analysis of $\mathrm{B}^{0} \rightarrow \mathrm{J} / \Psi \mathrm{K}^{* 0}$}

- The decay $(\mathrm{S} \rightarrow \mathrm{VV})$ can be decomposed as

- P-wave: $A_{0}$ (longitudial), $A_{\|}$(transverse-parallel), $A_{\perp}$ (transverse-perpendicular)

- S-wave: $A_{s}$ (non-resonant $K \pi$ )

- Strong phases: $\delta_{0}=0, \delta_{\|}, \delta_{\perp}$ and $\delta_{s}$

- Parity: even $\left(A_{0}, A_{\|}\right), \operatorname{odd}\left(A_{\perp}, A_{s}\right)$

- Probe BSM contributions,

which are approximately equal to

those in $\mathrm{B}_{\mathrm{s}} \rightarrow \mathrm{J} / \psi \varphi$

(golden mode for CPV in $\mathrm{B}_{\mathrm{s}}$ )

See talk by Olaf Steinkamp

- To measure the amplitudes the angular analysis is performed
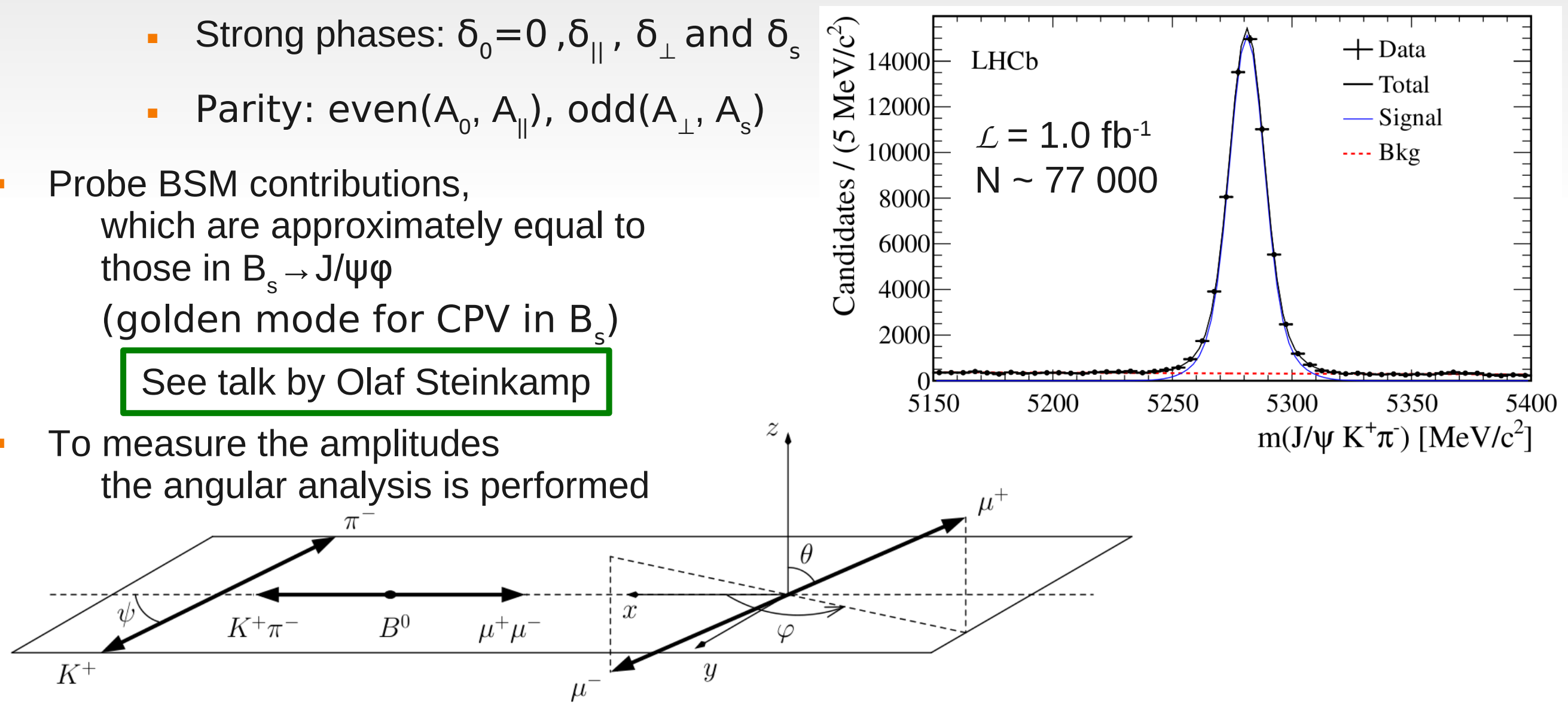


\section{Angular analysis of $\mathrm{B}^{0} \rightarrow \mathrm{J} / \Psi \mathrm{K}^{* 0}$}
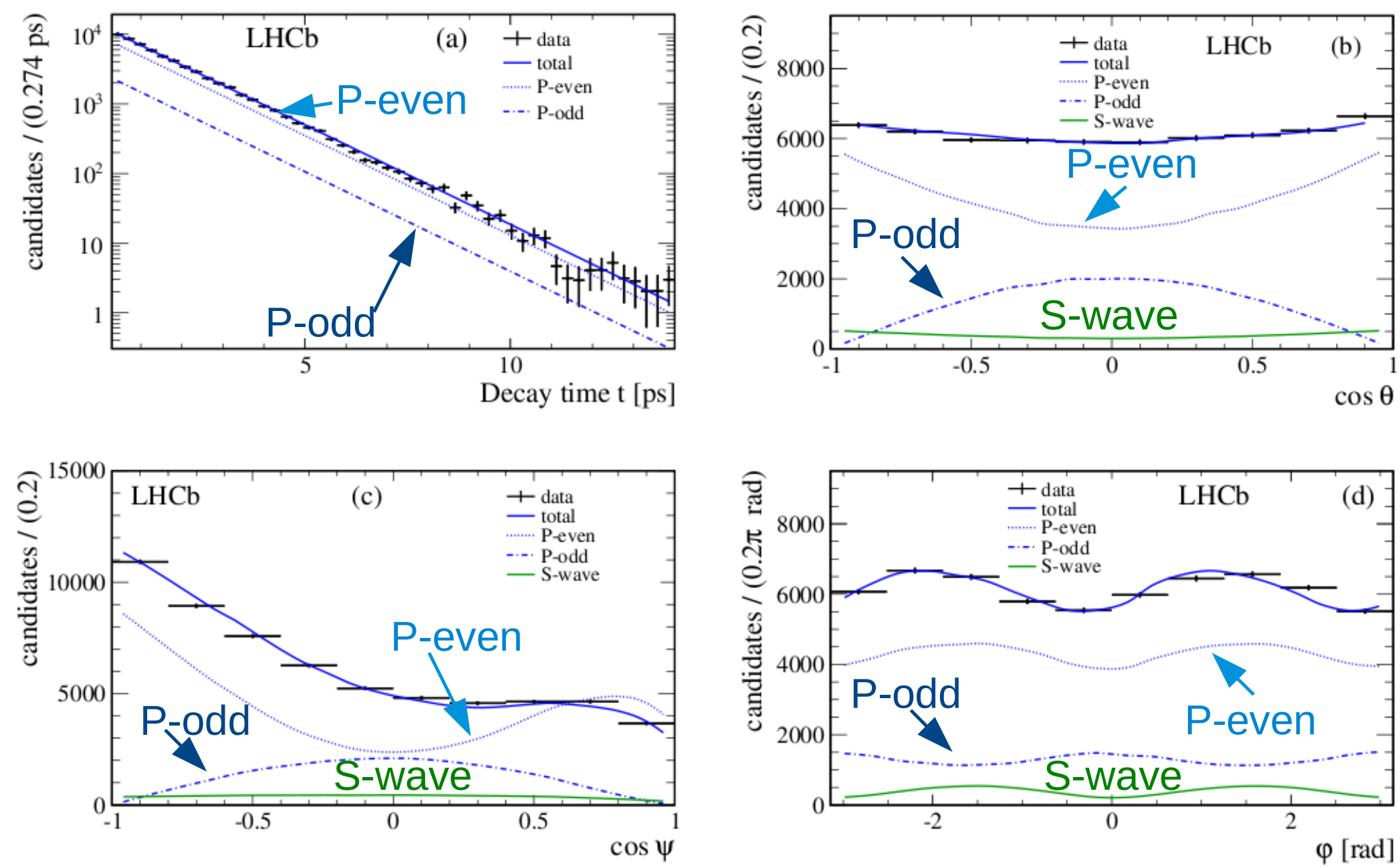

Dominant systematic unc.

- acceptance

- contribution from other resonances in $\mathrm{K} \pi$
$\left|A_{\|}\right|^{2}=0.227 \pm 0.004 \pm 0.011$, $\left|A_{\perp}\right|^{2}=0.201 \pm 0.004 \pm 0.008$ $\sigma_{\|}[\mathrm{rad}]=-2.94 \pm 0.02 \pm 0.03$, $\sigma_{\perp}[\mathrm{rad}]=2.94 \pm 0.02 \pm 0.02$
Consistent with previous measurements and SM predictions 
- Crucial role in CP violation studies [see talk of Olaf Steinkamp]

\& precise measurement of neutral B mixing parameters

- new possible channels for CPV studies in $\mathrm{B}^{0}{ }_{s}$

- Sensitive labarotary for electro-weak transition studies

- Direct probe of charmonium properties

- Study light quarks

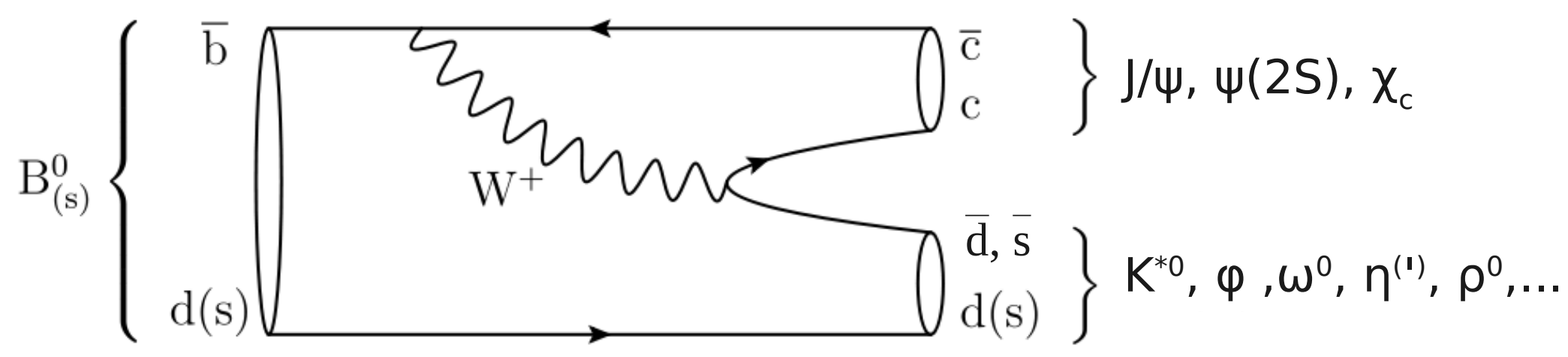


- $\quad \mathrm{B}_{\mathrm{s}}^{0} \rightarrow \mathrm{J} / \Psi \eta$ has been previously seen by Belle [PRL 108 (2012) 181808] and LHCb [NPB 867 (2013) 547]
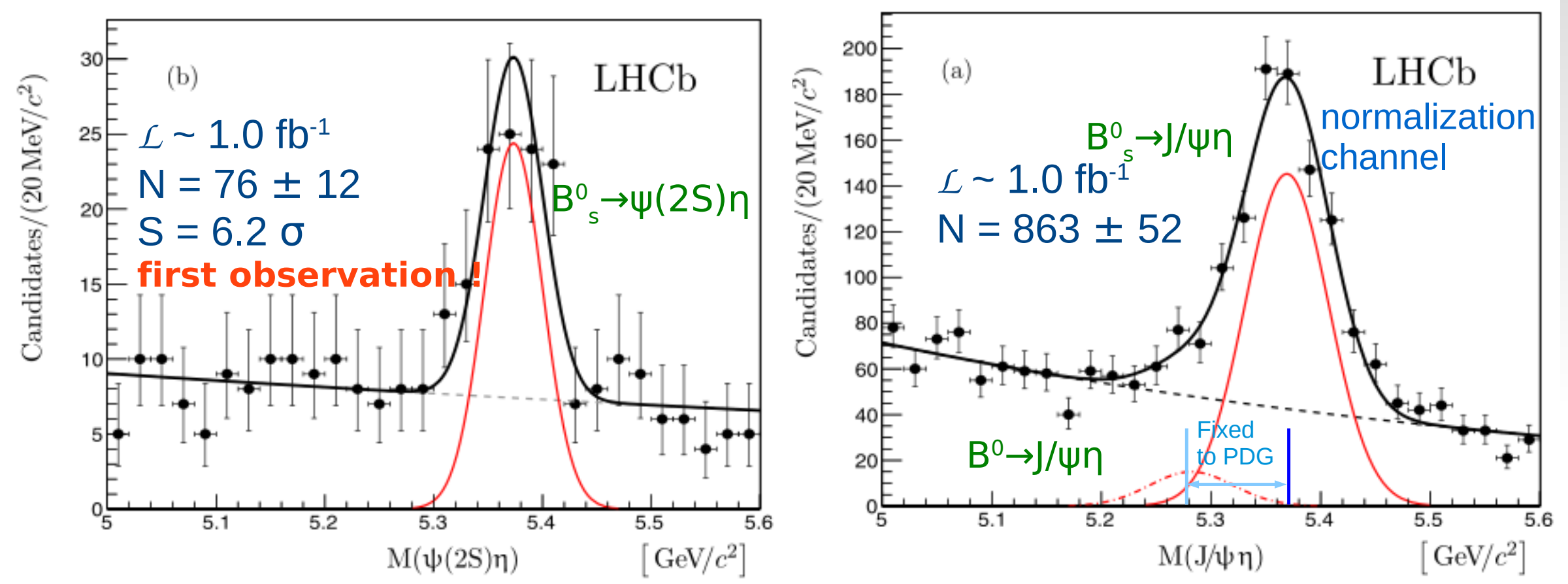

- Similar study for $\mathrm{B}^{0} \rightarrow \psi(2 S) \pi \pi$ and $\mathrm{B}^{0}{ }_{\mathrm{s}} \rightarrow \psi(2 S) \pi \pi$

- Explore intermediate resonance structure with sPlot

Dominant systematics:

efficiencies from simulation

$$
\frac{B R\left(B_{s}^{0} \rightarrow \psi(2 S) \eta\right)}{B R\left(B_{s}^{0} \rightarrow J / \psi \eta\right)}=0.83 \pm 0.14(\text { stat }) \pm 0.12(\text { syst }) \pm 0.02\left(B R_{\psi \rightarrow \mu^{+} \mu^{-}}\right)
$$


- In factorization approach $\mathrm{B} \rightarrow \mathrm{X}_{\mathrm{c} 0,2} \mathrm{~K}(\varphi)$ decay modes are expected to be suppressed with respect to $B \rightarrow X_{c 1} K(\varphi)$

- But the measured $\mathcal{B} R\left(\mathrm{~B}^{0} \rightarrow \mathrm{X}_{\mathrm{c0}} \mathrm{K}^{* 0}\right)=(1.7 \pm 0.3 \pm 0.2) \times 10^{-4}$ [BaBar, PR D78 (2008) 0911001] is compatible with $\mathcal{B R}\left(\mathrm{B}^{0} \rightarrow \mathrm{X}_{\mathrm{c1}} \mathrm{K}^{* 0}\right)=(2.5 \pm 0.2 \pm 0.2) \times 10^{-4}$ [BaBar, PRL 102 (2009) 132001] and $\quad \mathcal{B R}\left(\mathrm{B}^{0} \rightarrow \mathrm{X}_{\mathrm{c1}} \mathrm{K}^{* 0}\right)=\left(1.73_{-0.12-0.22}^{+0.15}\right) \times 10^{-4}$ [Belle, PR D78 (2008) 072004]

- While $\mathcal{B} R\left(\mathrm{~B}^{0} \rightarrow \mathrm{X}_{\mathrm{c} 2} \mathrm{~K}^{* 0}\right)=(6.6 \pm 1.8 \pm 0.5) \times 10^{-5}[\mathrm{BaBar}, \mathrm{PRL} 102$ (2009) 132001] can still be explained in factorization approach

- The $\mathrm{B}_{\mathrm{s}}^{0} \rightarrow \mathrm{X}_{\mathrm{c}} \varphi$ have not been previously observed

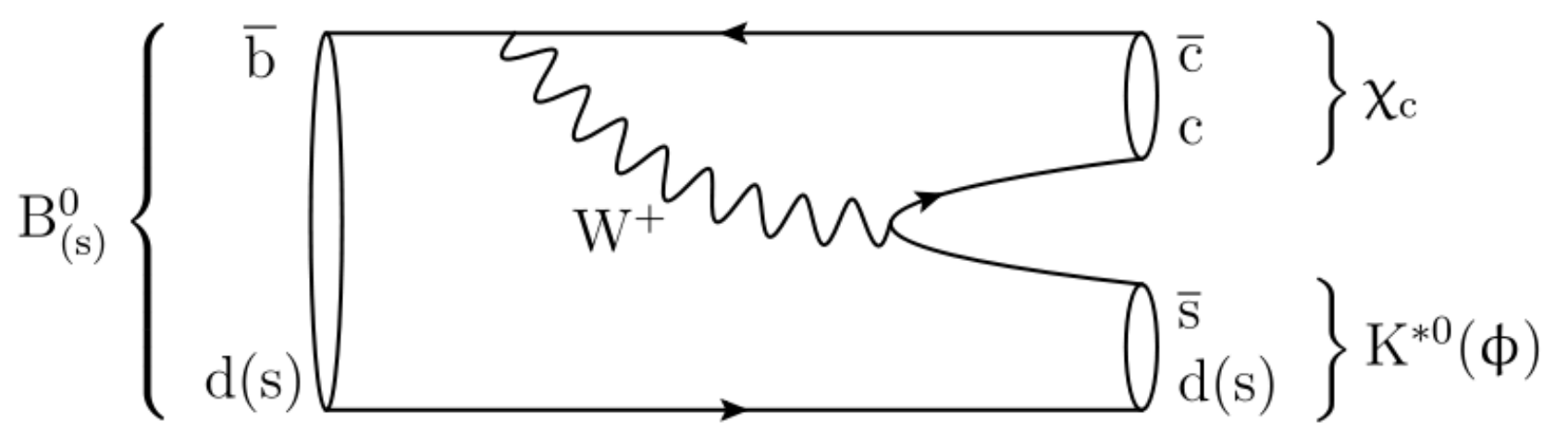



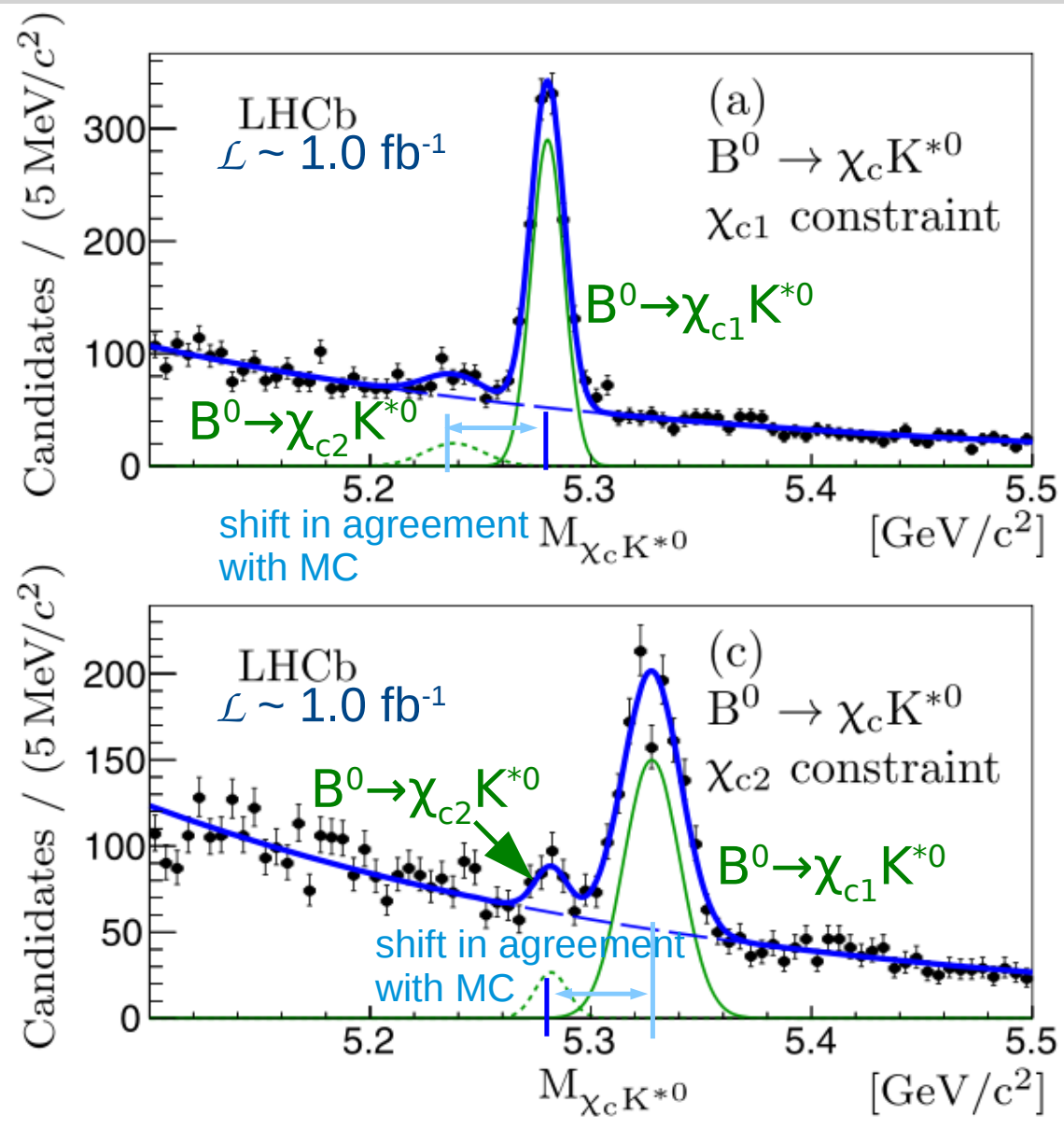

- Constraint J/ $\quad$ Y mass to $\chi_{\mathrm{c} 1,2}$ to resolve two peaks $\rightarrow$ mass shift in agreement with simulation

$$
\begin{aligned}
& \frac{B R\left(B^{0} \rightarrow \chi_{c 1} K^{* 0}\right)}{B R\left(B^{0} \rightarrow J / \psi K^{* 0}\right)}=\left(19.8 \pm 1.1(\text { stat }) \pm 1.2(\text { syst }) \pm 0.9\left(B R_{\chi_{c} \rightarrow J / \psi \gamma}\right)\right) \times 10^{-2} \\
& \frac{B R\left(B^{0} \rightarrow \chi_{c 2} K^{* 0}\right)}{B R\left(B^{0} \rightarrow \chi_{c 1} K^{* 0}\right)}=\left(17.1 \pm 5.0(\text { stat }) \pm 1.7(\text { syst }) \pm 1.1\left(B R_{\chi_{c} \rightarrow J / \psi \gamma}\right)\right) \times 10^{-2}
\end{aligned}
$$

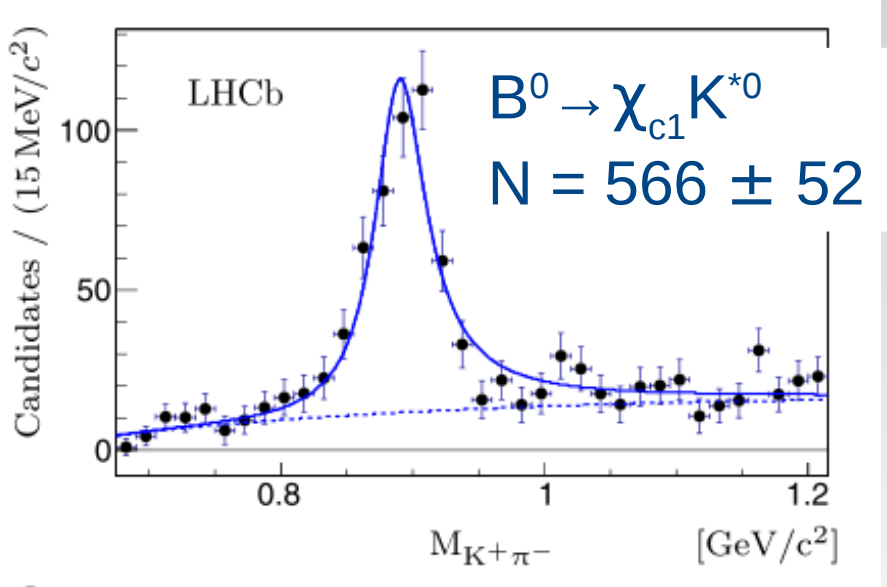

Background subtraction (sPlot)

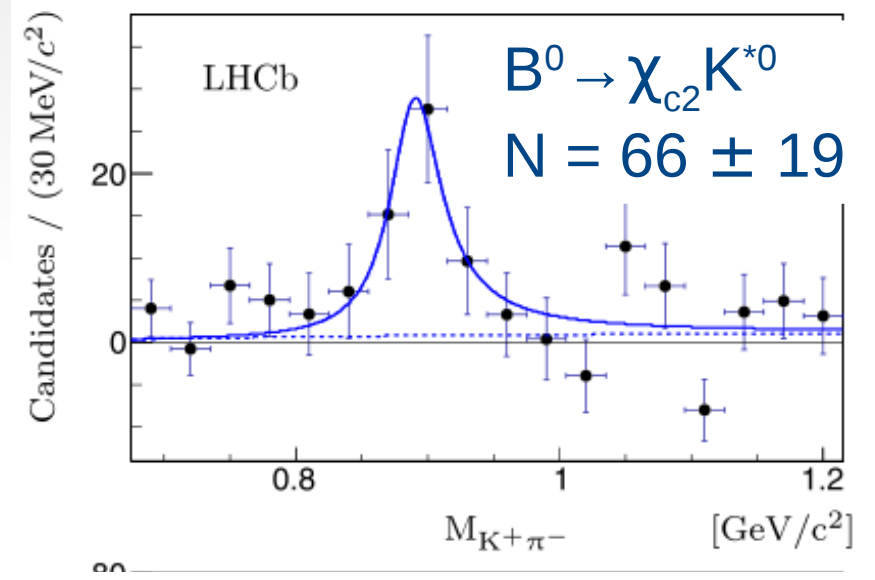

Dominant systematic:

- signal determination

- photon reconstruction

Compatible with previous measurements, but more precise

Nucl. Phys. B874 (2013) 663 


\section{Study of $\mathrm{B}_{\mathrm{s}}^{0} \rightarrow \mathrm{X}_{\mathrm{c} 1,2} \varphi$}

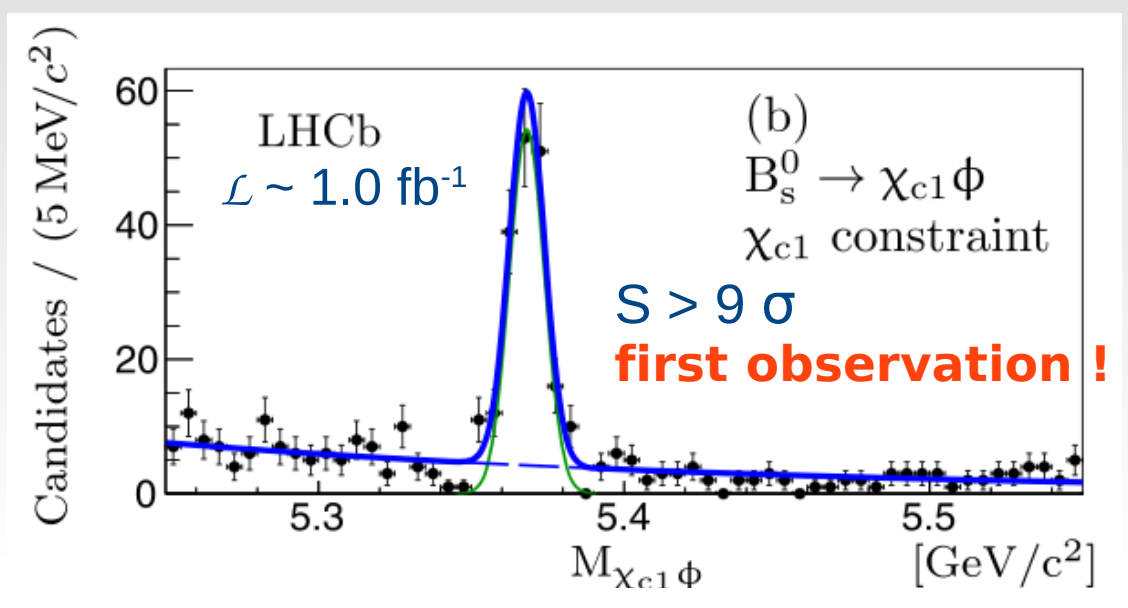

- Measure branching ratio relatively to $\mathrm{B}_{\mathrm{s}}^{0} \rightarrow \mathrm{J} / \Psi \varphi$

$$
\frac{B R\left(B_{s}^{0} \rightarrow \chi_{c 1} \phi\right)}{B R\left(B_{s}^{0} \rightarrow J / \psi \phi\right)}=\left(18.9 \pm 1.8(\text { stat }) \pm 1.3(\text { syst }) \pm 0.8\left(B R_{\chi_{c} \rightarrow J / \psi \gamma}\right)\right) \times 10^{-2}
$$

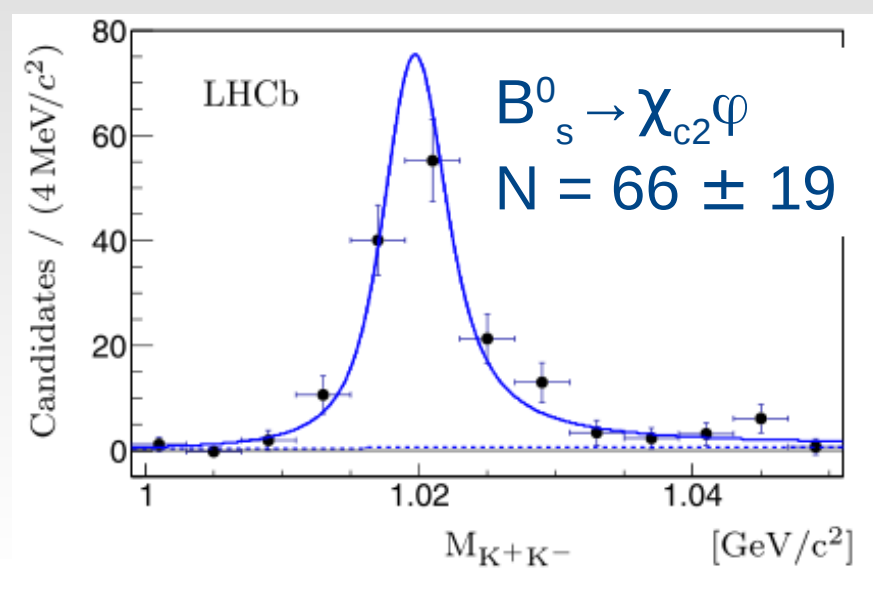

Dominant systematic:

- signal determination

- photon reconstruction 
X(3872) was discovered by Belle in 2003 [PRL 91 (2003) 262001], but it's nature still unclear

- C-parity $=+1$ since $X(3872) \rightarrow \mathrm{J} / \Psi \mathrm{Y}$ is observed

- CDF excluded all JPC combinations except $1^{++}$and $2^{++}$[PRL 98 (2007) 132002]

- BaBar favoured $2^{-+}(68 \% \mathrm{CL})$ by studying $\mathrm{X}(3872) \rightarrow \mathrm{J} / \psi \omega^{0}$, but not ruled out $1^{++}(7 \% \mathrm{CL})$ [PRD 82 (2010) 011101]

- Belle couldn't distinguish $1^{++}$and $2^{-+}$by analysing 1D distributions [PRD 84 (2011) 052004]

Determination of quantum numbers is crucial for the interpretation of the state

- $1^{++}:$molecular, tetraquark, $\chi_{c}\left(2^{3} \mathrm{P}_{1}\right)$ ?

- $2^{-+}: \eta_{c}\left(1^{1} D_{2}\right) ?$ 


\section{X(3872) quantum numbers}

5-D angular analysis of $\mathrm{B}^{+} \rightarrow \mathrm{X}(3872) \mathrm{K}^{+}$, $\mathrm{X}(3872) \rightarrow \mathrm{J} / \psi \pi^{+} \pi^{-}$

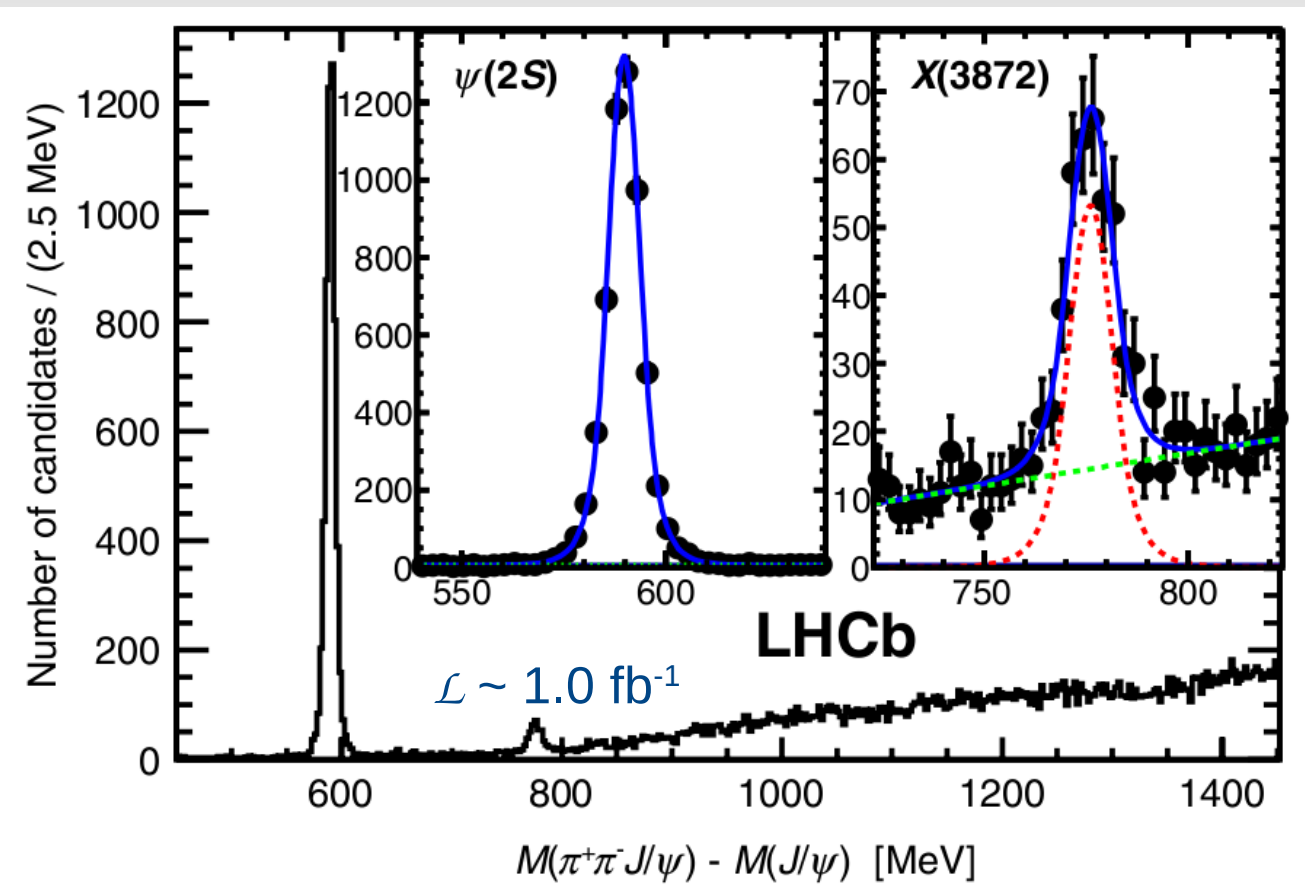

$$
\begin{aligned}
& \mathrm{B}^{+} \rightarrow \Psi(2 \mathrm{~S}) \mathrm{K}^{+}, \quad \mathrm{N}=5642 \pm 76 \\
& \mathrm{~B}^{+} \rightarrow \mathrm{X}(3872) \mathrm{K}^{+}, \mathrm{N}=313 \pm 26
\end{aligned}
$$

- Angular distributions carry information about $\mathrm{J}^{\mathrm{PC}}$
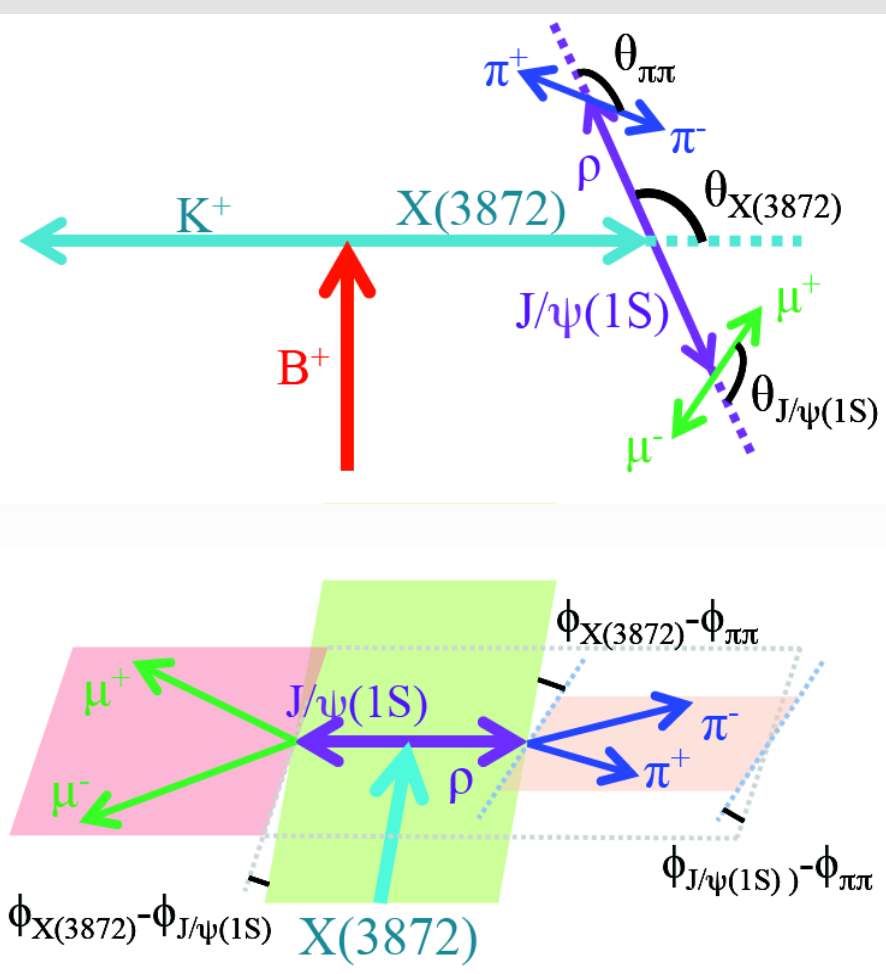


\section{X(3872) quantum numbers}

- Likelihood-ratio test to discriminate between to hypothesis

Angular correlations magnify differences between spin hypothesis:

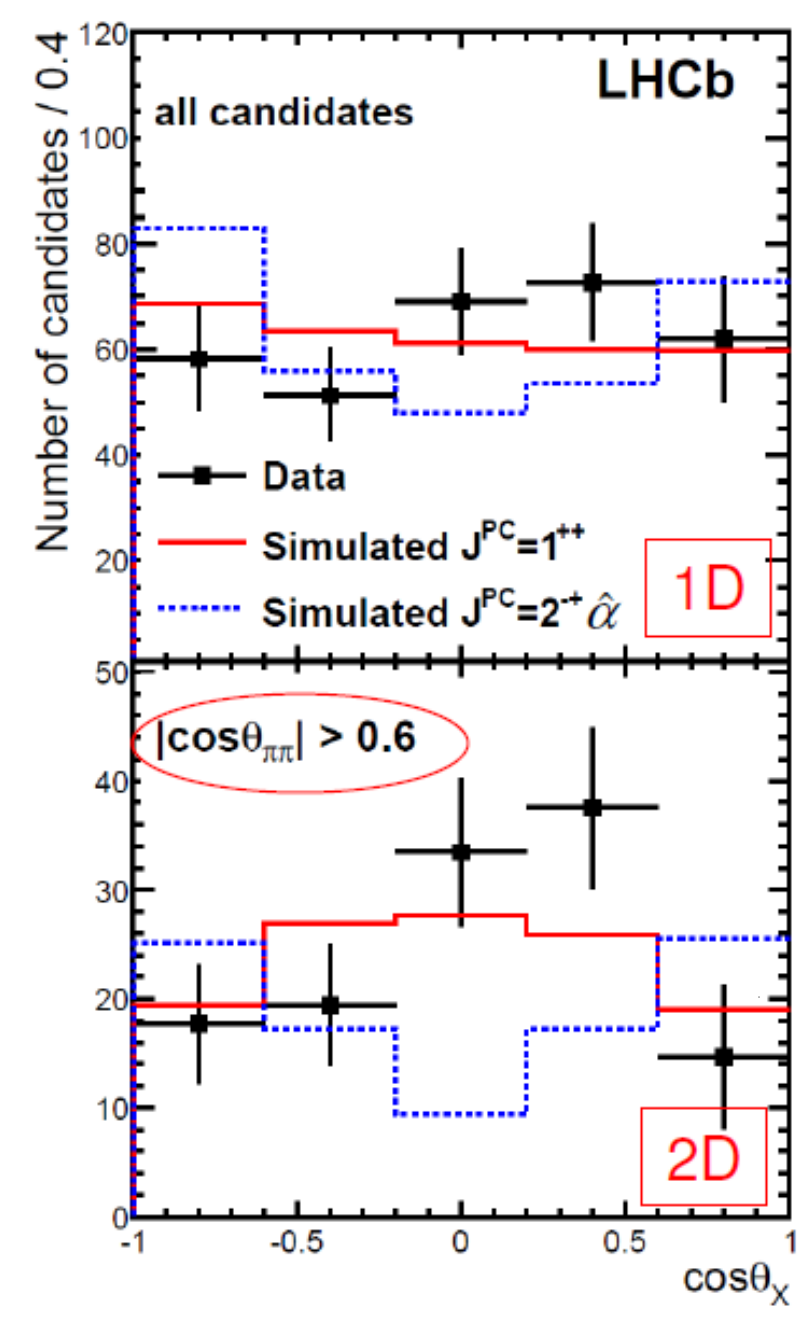$$
\mathrm{t}=-2 \ln \left[\mathcal{L}\left(2^{-+}\right) / \mathcal{L}\left(1^{++}\right)\right]
$$

- Compare with simulated statistics

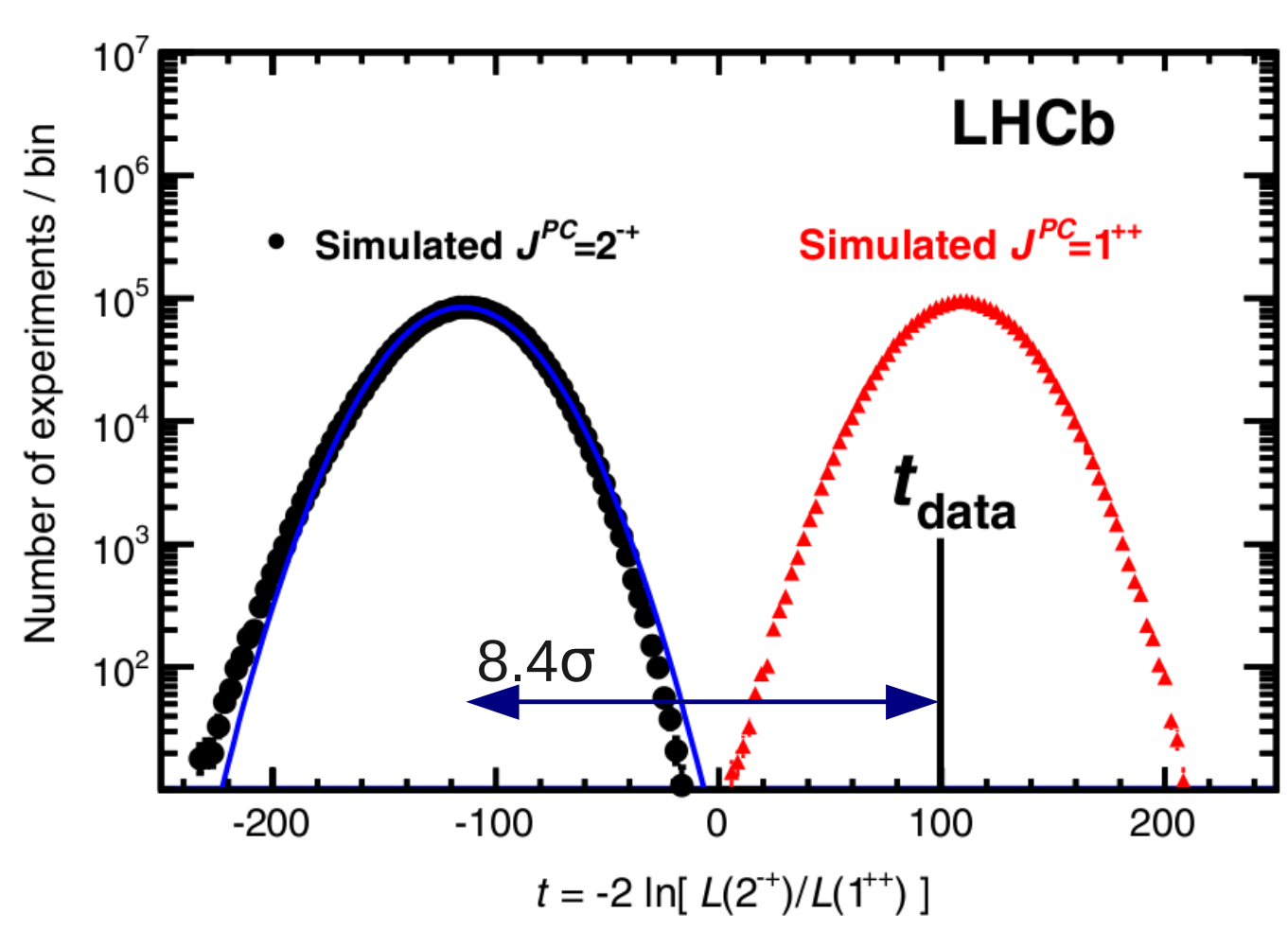

Result:

$2^{-+}$is rejected with $8.4 \sigma$, with $p$-value for $1^{++}$of $34 \%$ 
- The $B_{c}$ meson, composed of two heavy quarks $(b \bar{c})$, is a unique, being the only weak decaying heavy quarkonium system

- Prior to LHCb only the $\mathrm{B}_{c}^{+} \rightarrow \mathrm{J} / \psi \pi^{+}$and $\mathrm{B}_{\mathrm{c}}^{+} \rightarrow \mathrm{J} / \psi \mu^{+} \nu_{\mu}$ decays were observed

- At LHCb $f\left(\bar{b} \rightarrow B_{c}^{+}\right) \sim 1 / 1000$

- LHCb has already observed new decay mode:

$\mathrm{B}_{\mathrm{c}}^{+} \rightarrow \mathrm{J} / \psi \pi^{+} \Pi^{-} \pi^{+}[\mathrm{PRL} 108$ (2012) 251802]

- Four more new decay modes in this summer $\rightarrow$ see next 
Test different theoretical models for $\mathrm{B}_{\mathrm{c}}^{+}$ decays

Analysis strategy:

- multivariate selection (BDT) to supress background

- $\mathrm{B}_{\mathrm{c}}^{+} \rightarrow \mathrm{J} / \Psi \pi^{+}$as normalization channel

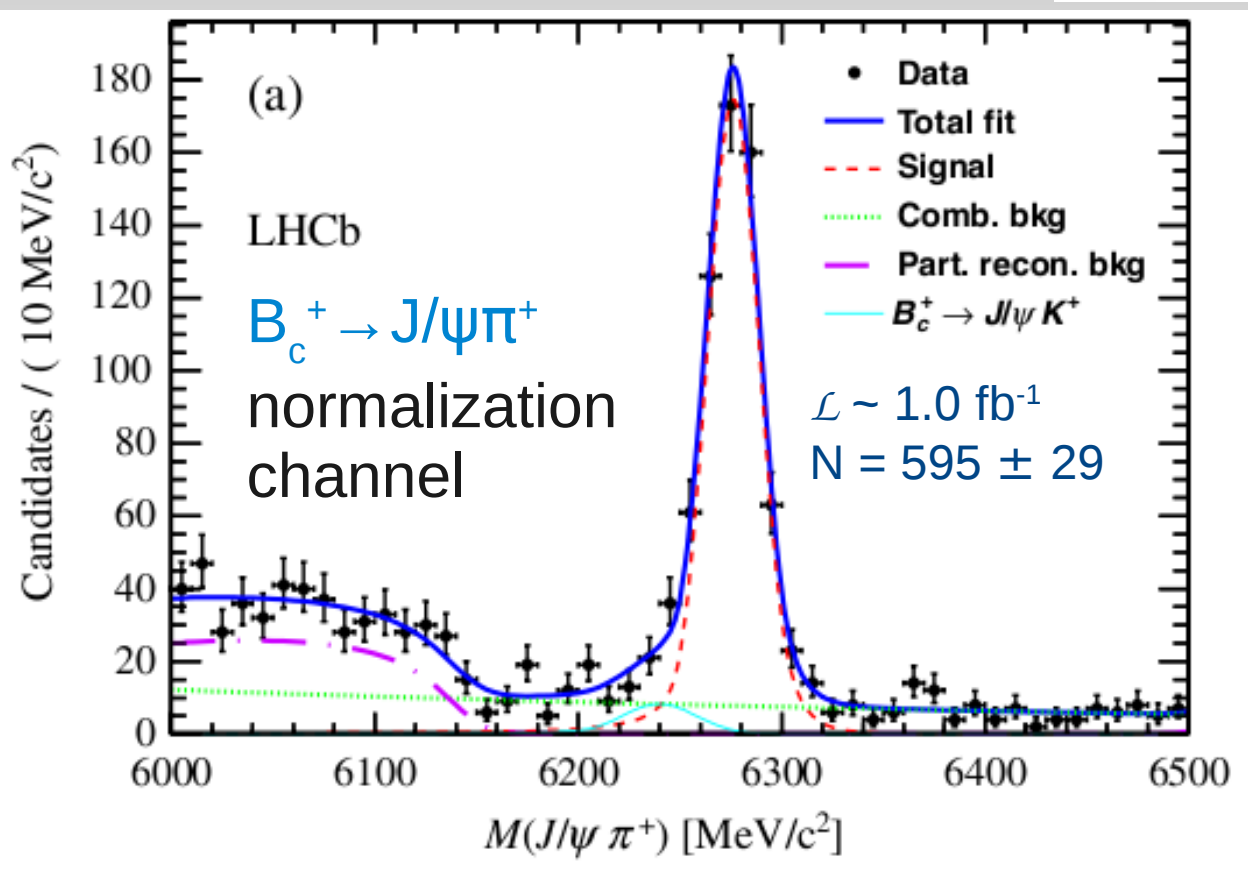

- Dominant syst:

- understanding of the BDT selection

- background shape

$B R\left(B_{c}^{+} \rightarrow \psi(2 S) \pi^{+}\right) / B R\left(B_{c}^{+} \rightarrow J / \psi \pi^{+}\right)=$ $0.250 \pm 0.068$ (stat $) \pm 0.014$ (syst) $\pm 0.006(B R)$

- The result favours relativistic quark model prediction with respect to the others

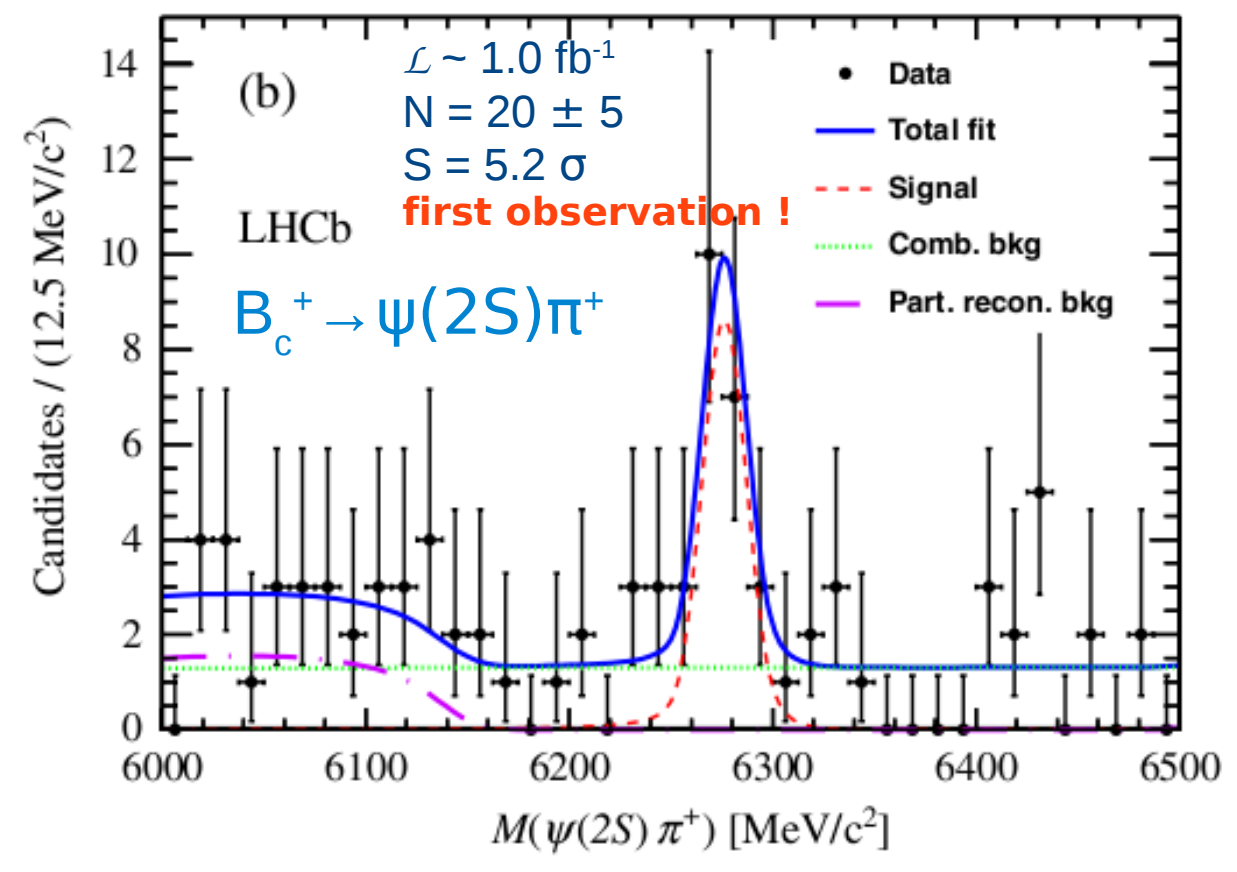


- The branching fraction relative to $\mathrm{B}_{\mathrm{c}}{ }^{+} \rightarrow \mathrm{J} / \psi \pi^{+}$is measured

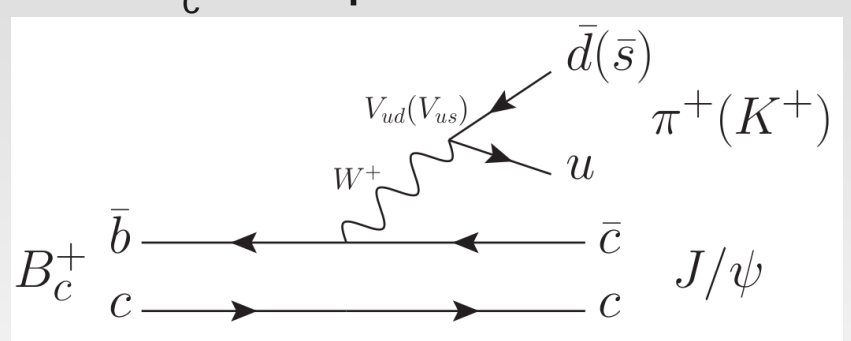

- Naive expectation:

$$
\frac{\mathcal{B}\left(B_{c}^{+} \rightarrow J / \psi K^{+}\right)}{\mathcal{B}\left(B_{c}^{+} \rightarrow J / \psi \pi^{+}\right)} \approx\left|\frac{V_{u s} f_{K^{+}}}{V_{u d} f_{\pi^{+}}}\right|^{2}=0.077
$$

- Theoretical predictions lie within

$0.054-0.088$ range

$\rightarrow$ test hadronization model

- Analysis strategy:

- Multivariate selection (BDT)

- bins of discriminating $\mathrm{K}-\pi$ identification variable

pion-like
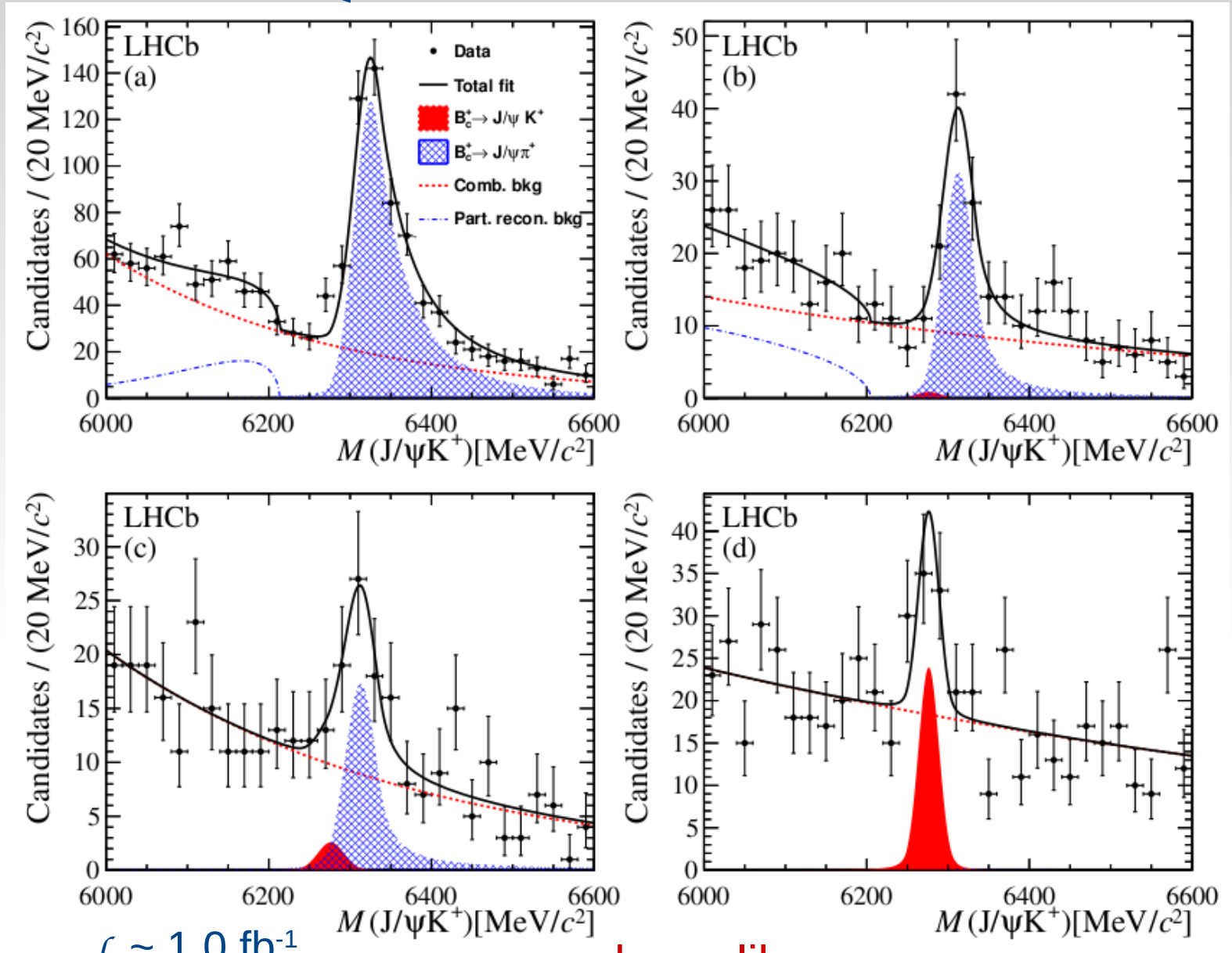

$\mathcal{L} \sim 1.0 \mathrm{fb}^{-1}$

kaon-like

$\mathrm{N}=46 \pm 14$

$\mathrm{S}=5.0 \sigma$

first observation !

$$
\frac{B R\left(B_{c}^{+} \rightarrow J / \psi K^{+}\right)}{B R\left(B_{c}^{+} \rightarrow J / \psi \pi^{+}\right)}=0.069 \pm 0.019 \pm 0.005
$$

Dominant systematic:

- understanding of the BDT selection 
The branching fraction relative to $\mathrm{B}_{\mathrm{c}}^{+} \rightarrow \mathrm{J} / \psi \pi^{+}$is measured

Three leading contributions

Test assumption that

spectator one dominates \& factorization holds

Low energy release allows precision mass measurement

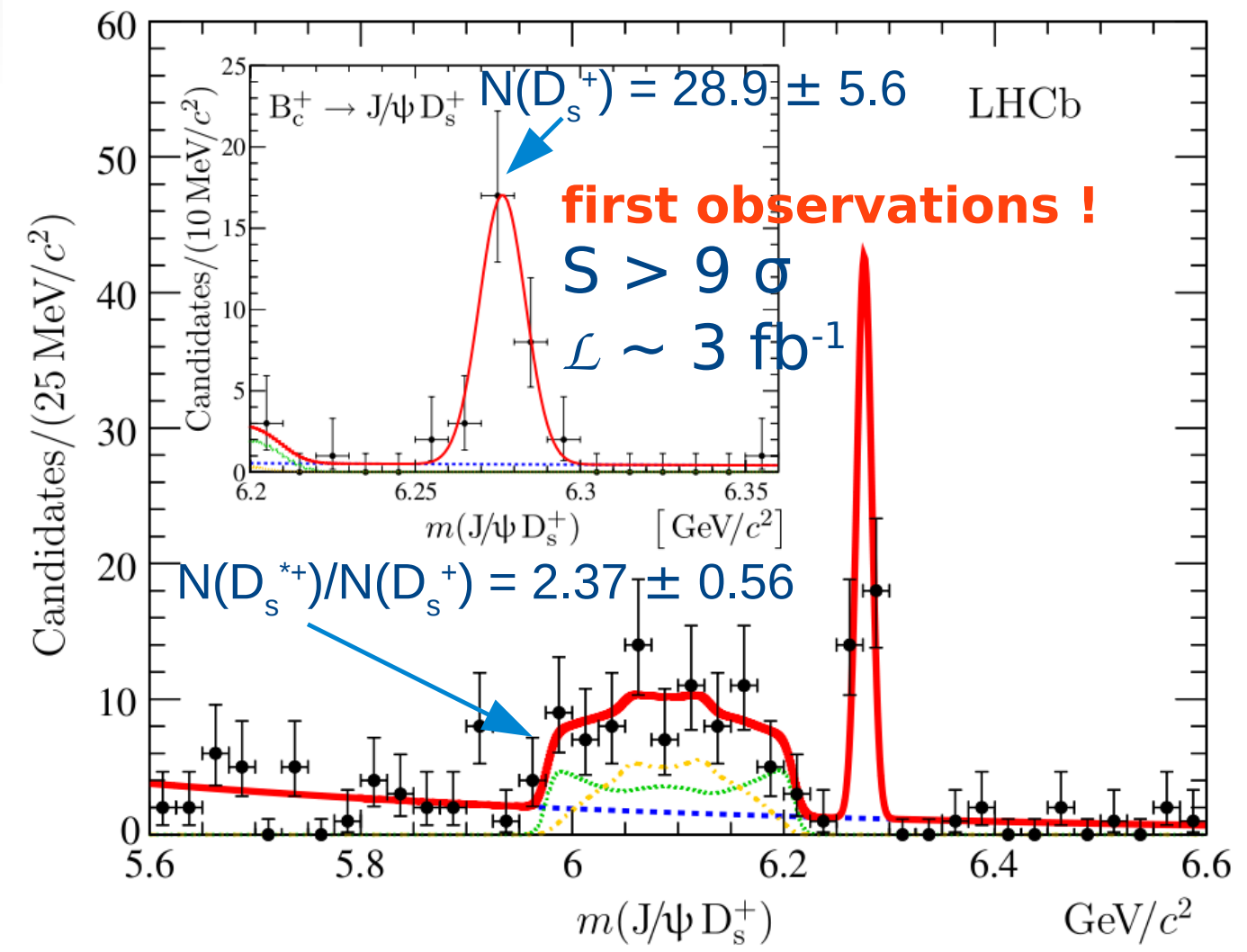

$$
\begin{aligned}
& \frac{B R\left(B_{c}^{+} \rightarrow J / \psi D_{s}^{+}\right)}{B R\left(B_{c}^{+} \rightarrow J / \psi \pi^{+}\right)}=2.90 \pm 0.57(\text { stat }) \pm 0.24(\text { syst }) \\
& \left.\frac{B R\left(B_{c}^{+} \rightarrow J / \psi D_{s}^{*+}\right)}{B R\left(B_{c}^{+} \rightarrow J / \psi D_{s}^{+}\right)}=2.37 \pm 0.56(\text { stat }) \pm 0.10 \text { ( syst }\right)
\end{aligned}
$$

- Compatible with naive expectations from $B \rightarrow D^{\star} D_{s}^{(*)+}\left(\pi^{+}\right)$decays

$$
m_{B_{c}^{+}}=6276.28 \pm 1.44 \text { (stat) } \pm 0.36 \text { ( syst) } M e V / c^{2}
$$

- In agreement with PDG (2013 update): $\mathrm{m}\left(\mathrm{B}_{\mathrm{c}}^{+}\right)=6274.5 \pm 1.8 \mathrm{MeV} / \mathrm{c}^{2}$

$$
m\left(\mathrm{~J} / \psi \mathrm{D}_{\mathrm{s}}^{+}\right)
$$


LHCb shows excellent performance in measuring $B$ decays to charmonia

- Provide input for measurements of CPV and mixing in B mesons

- Explore charmonium properties

- Serie of new decays observed $\left(\mathrm{B}_{\mathrm{s}}^{0} \rightarrow \Psi(2 \mathrm{~S}) \eta, \mathrm{B}_{\mathrm{s}}^{0} \rightarrow \mathrm{X}_{\mathrm{c} 1} \varphi, \mathrm{B}_{\mathrm{c}}^{+} \rightarrow \ldots\right)$

- $\quad X(3872)$ quantum numbers determined to be $1^{++}$

- Study of properties and observe new decays of the $B_{c}{ }^{+}$meson

$\left(B_{c}^{+} \rightarrow J / \psi K^{+}, B_{c}^{+} \rightarrow J / \psi D_{s}^{(*)}\right)$

- Plus many other analysis, for instance

- Precision measurement of $\wedge_{b}$ lifetime [arXiv:1306.6723]

- First upper limits on $\mathcal{B R}\left(\mathrm{B}^{0}{ }_{\mathrm{s}} \rightarrow \mathrm{J} / \Psi \mathrm{pp}\right)$ and $\mathcal{B R}\left(\mathrm{B}^{+} \rightarrow \mathrm{J} / \Psi \mathrm{pp} \pi^{+}\right)$[arXiv: 1306.4489]

Thank you! 


\section{Backup}


- According to Hevay Quark Expansion model $\tau\left(\Lambda_{b}\right) / \tau(B) \simeq 1$ with difference only a few persent expected

- Average experimental give
$0.798 \pm 0.052$ [arXiv:hep-ph/0304132]
$0.786 \pm 0.034$ [arXiv:hep-ph/0310241, arXiv:hep-ph/0203089]

- Precision measurement is necessary

- Strategy:

- Use similar decay modes $\Lambda_{b} \rightarrow J / \psi p K^{-}$and $B \rightarrow J / \psi \pi^{+} K^{-} \rightarrow$ systematic uncertainty cancels in the ratio

- BDT

- Sideband

to subtract background

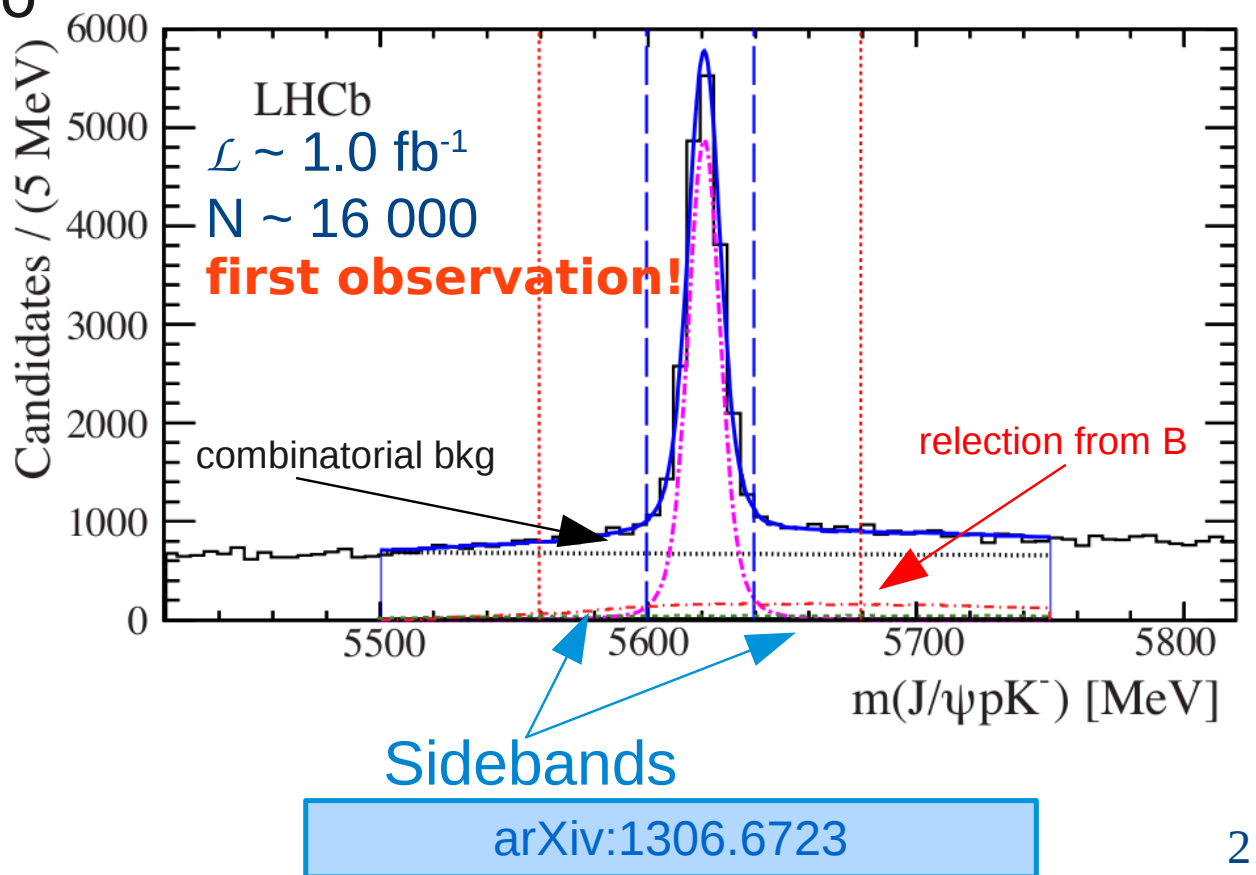


- Use whole pK mass region

- Decay time acceptance from simulation

weighted to match $\mathrm{pK}(\pi \mathrm{K})$ mass distribution

- Determine $\Delta_{\Lambda B}=\frac{1}{\tau_{\Lambda}}-\frac{1}{\tau_{B}} \quad$ from ratio of yields

- Dominant uncertainty:

- decay time fit range

- acceptance slope

$$
\begin{aligned}
& \frac{\tau_{\Lambda_{b}^{0}}}{\tau_{\bar{B}^{0}}}=\frac{1}{1+\tau_{\bar{B}^{0}} \Delta_{\Lambda B}}=0.976 \pm 0.012 \pm 0.006 \\
& \tau_{\Lambda_{b}^{0}}=1.482 \pm 0.018 \pm 0.012 \mathrm{ps}
\end{aligned}
$$

- In agreement with HQE expectation of $\tau\left(\wedge_{b}\right) / \tau(B)=1$

- Consistent with, but more precise than,

current world average: $\tau\left(\Lambda_{b}\right)=1.429 \pm 0.024$ ps

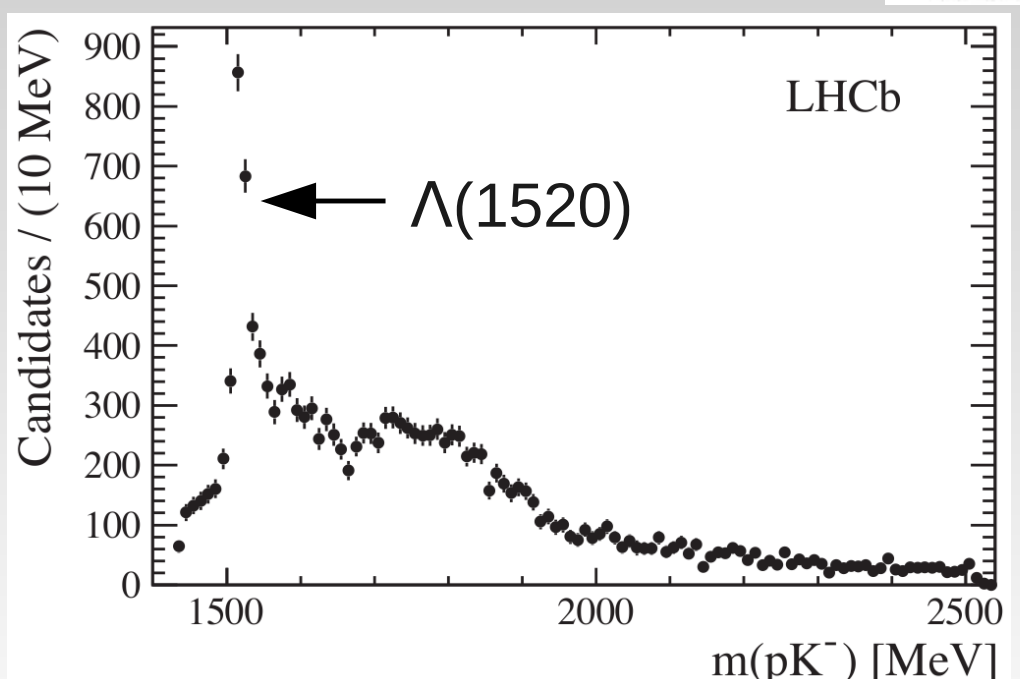

$\mathrm{m}\left(\mathrm{pK}^{-}\right)[\mathrm{MeV}]$

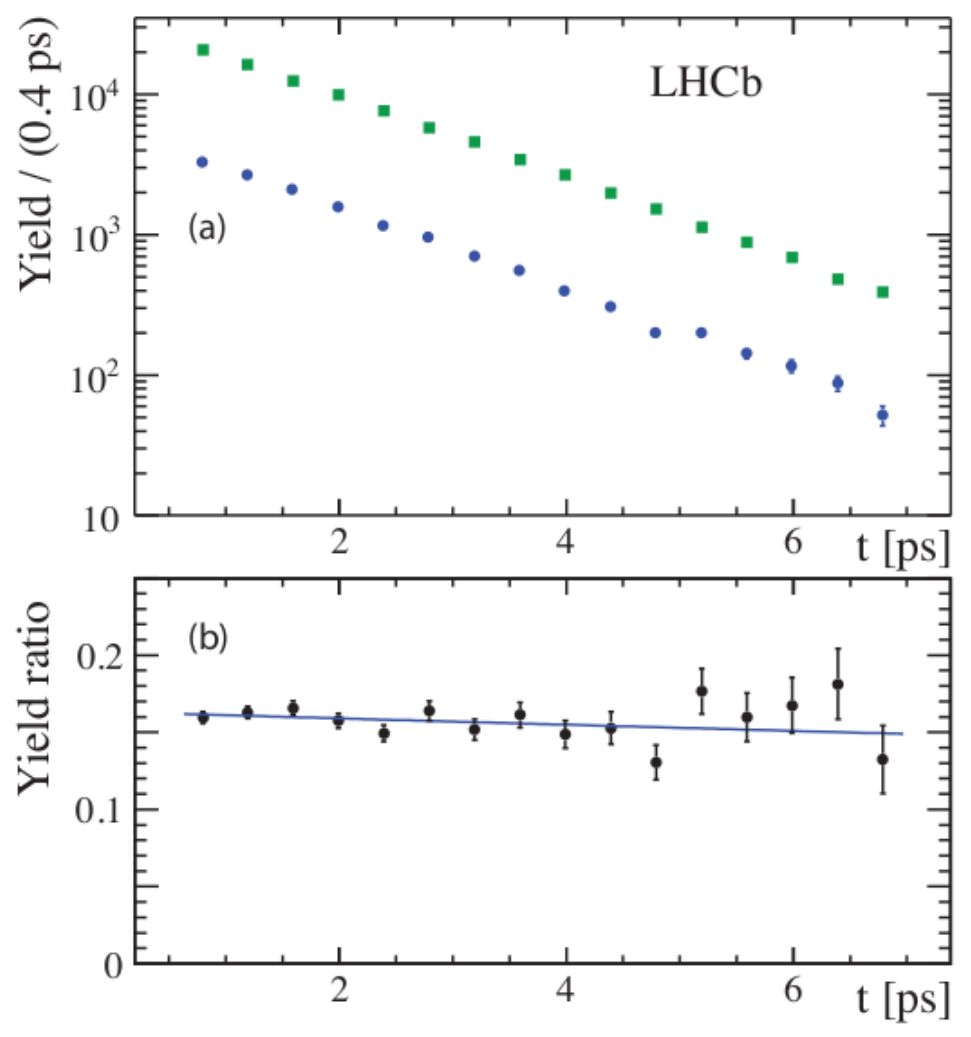

arXiv:1306.6723 
- The study of such decays has not been extensively explored mainly due to the suppressed branching fractions of typically $\mathrm{O}\left(<10^{-5}\right)$

- Dibaryon production in $B$ meson decays has been studied in decays $B^{+} \rightarrow K^{+} p \bar{p}$ or $B^{0} \rightarrow \bar{D}^{0} p \bar{p}$

- Branching fraction is $\sim 10 \%$ that of the corresponding decay with pp replaced by $\pi^{+} \pi^{-}$

- In contrast

$$
\begin{aligned}
B R\left(B^{0} \rightarrow J / \psi \pi^{+} \pi^{-}\right) & =(4.6 \pm 0.9) \times 10^{-5} \\
B R\left(B^{0} \rightarrow J / \psi p \bar{p}\right) & <8.3 \times 10^{-7}
\end{aligned}
$$




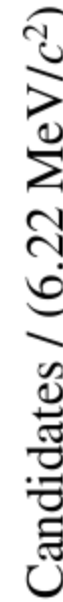
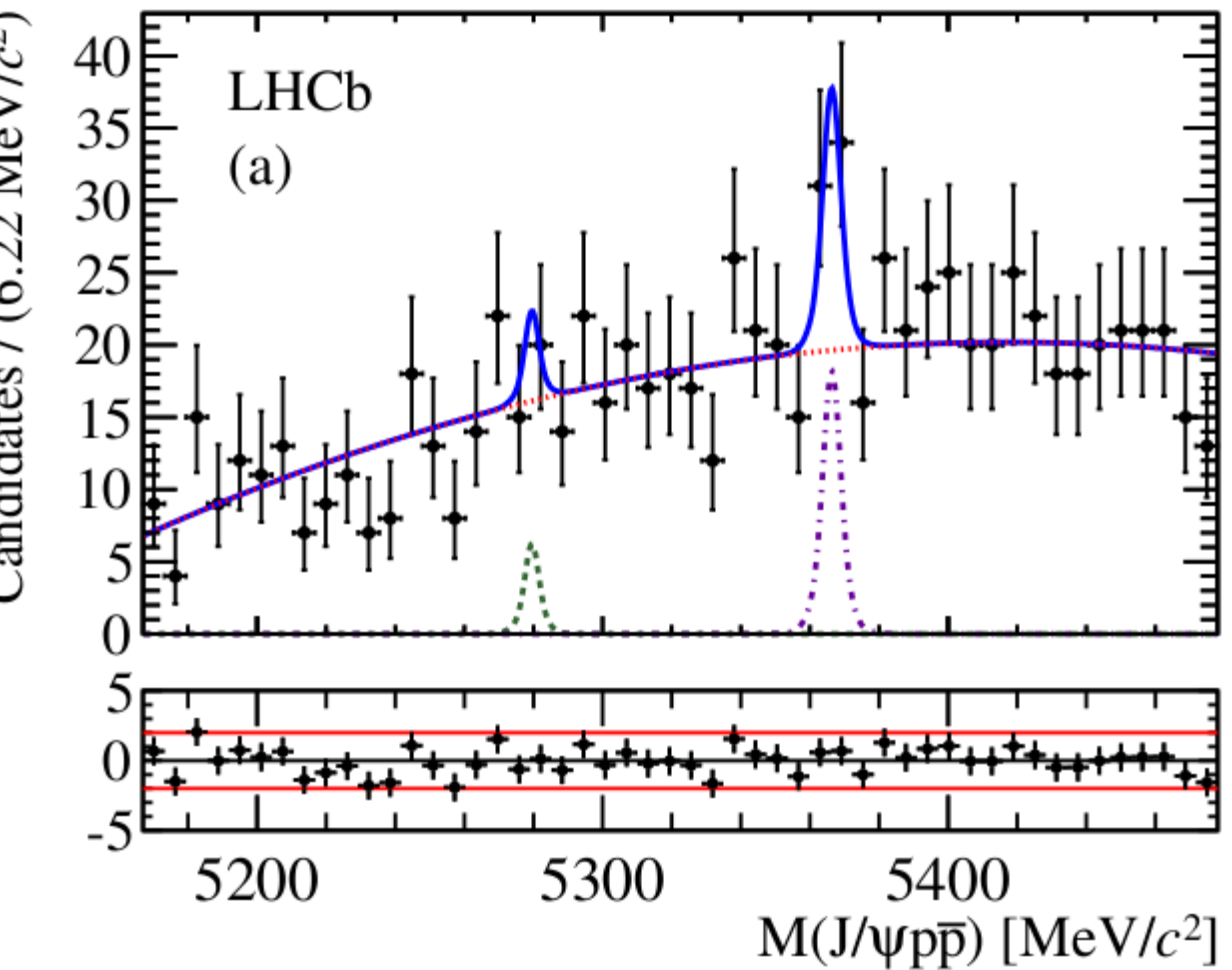

No significant signals are seen
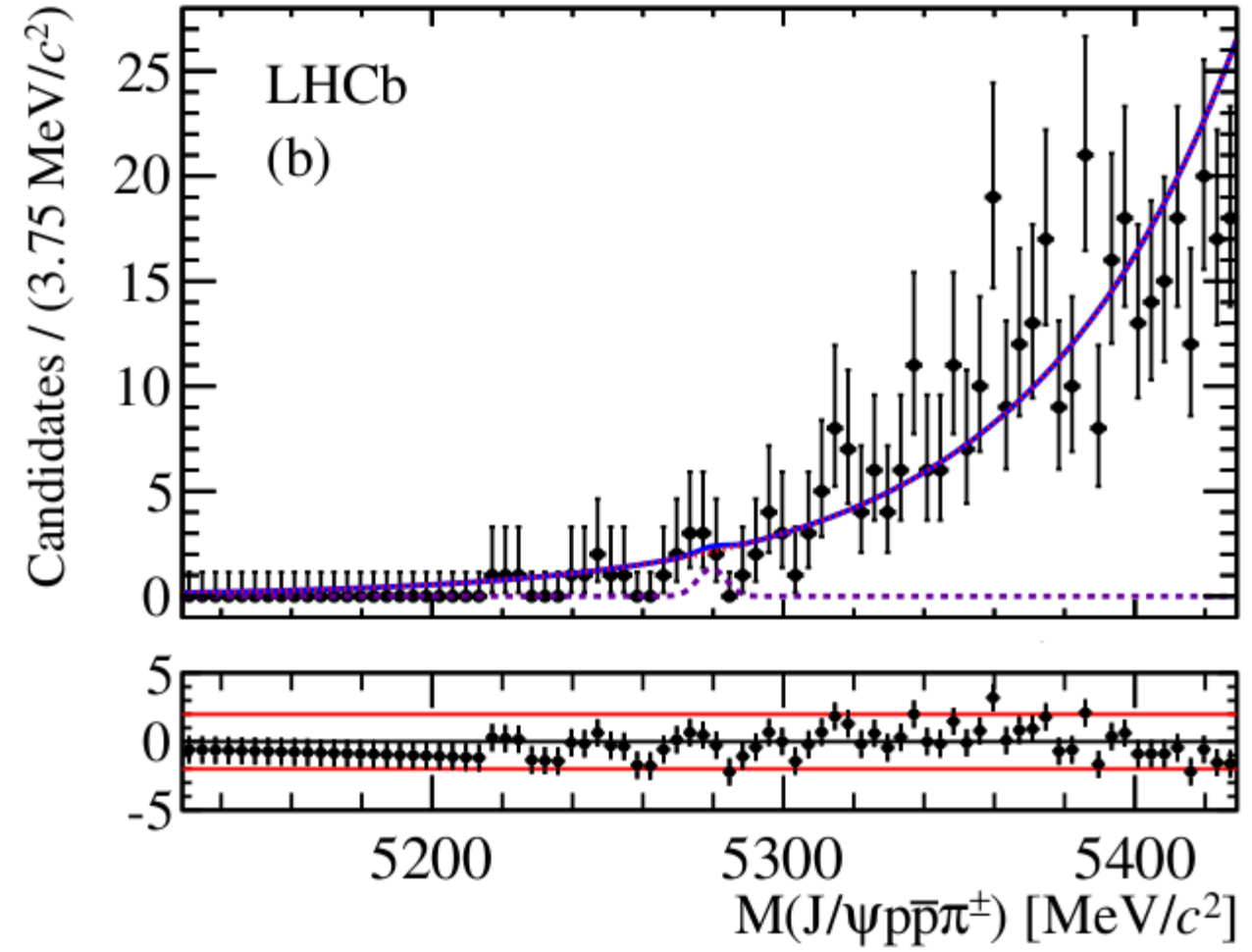

$$
\left.\begin{array}{lll}
B R\left(B^{0} \rightarrow J / \psi p \bar{p}\right) & <5.2(6.0) \times 10^{-7} & @ 90 \%(95 \%) C L \\
B R\left(B_{s}^{0} \rightarrow J / \psi p \bar{p}\right) & <4.8(5.3) \times 10^{-6} & @ 90 \%(95 \%) C L \\
B R\left(B^{+} \rightarrow J / \psi p \bar{p} \pi^{+}\right) & <5.0(6.1) \times 10^{-7} & @ 90 \%(95 \%) C L
\end{array}\right\} \text { first limits! }
$$



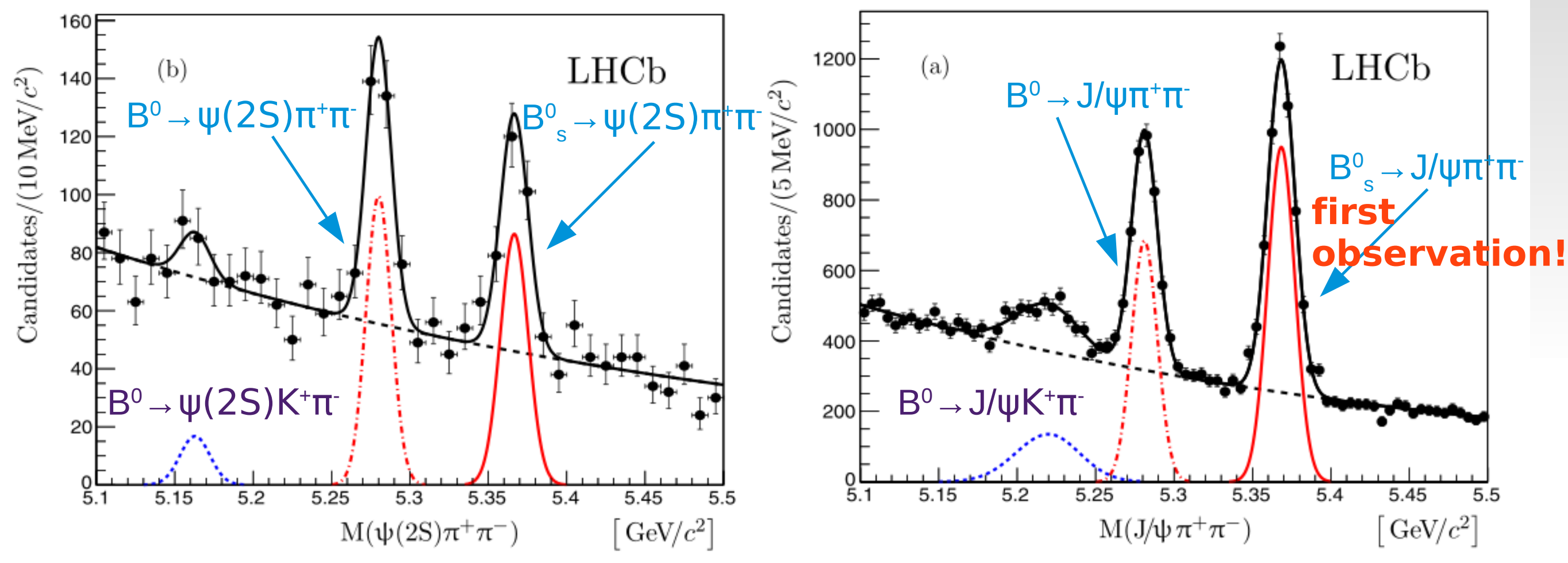

$\frac{B R\left(B^{0} \rightarrow \psi(2 S) \pi^{+} \pi^{-}\right)}{B R\left(B^{0} \rightarrow J / \psi \pi^{+} \pi^{-}\right)}=0.56 \pm 0.07($ stat $) \pm 0.05($ syst $) \pm 0.01\left(B R_{\psi \rightarrow \mu^{+} \mu^{-}}\right)$
$\frac{B R\left(B_{s}^{0} \rightarrow \psi(2 S) \pi^{+} \pi^{-}\right)}{B R\left(B_{s}^{0} \rightarrow J / \psi \pi^{+} \pi^{-}\right)}=0.34 \pm 0.04($ stat $) \pm 0.03($ syst $) \pm 0.01\left(B R_{\psi \rightarrow \mu^{+} \mu^{-}}\right)$

